\title{
Compósitos cimentícios reforçados com fibras de eucalipto puras e tratadas com tetraetilortossilicato (TEOS 98\%)
}

\author{
Cementitious composites strengthened with pure \\ eucalyptus fiber and treated with tetraethyl ortosilicate \\ (TEOS 98\%)
}

\section{Alessandra Buss Tessaro \\ Margarete Regina Freitas Gonçalves \\ Darci Alberto Gatto \\ Edilson Nunes Pollnow \\ Neftali Lenin Villarreal Carreño \\ Rafael de Ávila Delucis}

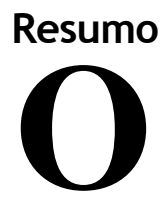

presente trabalho apresenta a obtenção de maior flexibilidade e elasticidade de uma matriz de cimento Portland de alta resistência inicial (CPV) adquirida a partir da adição de fibras de eucalipto puras e tratadas com tetraetilortossilicato TEOS $98 \%$. As fibras utilizadas foram caracterizadas quanto à morfologia, através de microscopia óptica e com o emprego do software Anatomic MIPro, e quanto à absorção de água, para a verificação da eficiência da impregnação do TEOS 98\%. Para a obtenção dos compósitos foram formuladas massas de cimento com adição de fibras de eucalipto puras e tratadas nos teores de 2\%, 5\% e 10\% em massa. Os compósitos foram caracterizados quanto a absorção de água, porosidade aparente e módulo de elasticidade (MOE) a partir da resistência à flexão a três pontos. A análise foi comparativa entre os compósitos de cimento-fibras de eucalipto e uma massa pura de cimento Portland de alta resistência inicial. Os resultados mostraram que os maiores valores de reforço foram obtidos no compósito aditivado com $2 \%$ de fibra de eucalipto tratada com TEOS $98 \%$, visto que neste se obtiveram os maiores MOE, caracterizando maior controle de fissuras e de absorção de energia após a fratura da matriz de cimento.

Margarete Regina Freitas Gonçalves Universidade Federal de Pelotas Pelotas - RS - Brasil

Darci Alberto Gatto Universidade Federal de Pelotas Pelotas - RS - Brasil

Edilson Nunes Pollnow Universidade Federal do Rio Grande Rio Grande - RS - Brasil

Neftali Lenin Villarreal Carreño Universidade Federal de Pelotas Pelotas - RS - Brasil

Rafael de Ávila Delucis Universidade Federal de Pelotas Pelotas - RS - Brasil

Recebido em 04/10/14 Aceito em 18/04/15
Palavras-chaves: Fibras de eucalipto. Tratamento superficial. Compósito cimentofibras. Propriedades físicas. Módulo de elasticidade.

\section{Abstract}

This paper presents the attainment of higher flexibility and elasticity in a high initial strength (CPV) Portland cement matrix, achieved through the addition of pure eucalyptus fibers treated with tetraethylorthosilicate TEOS $98 \%$. The fibers used were characterized by their morphology through optical microscopy and through the use of anatomic MIPRO software; its water absorption properties were analysed using the TEOS 98\% impregnation efficiency test. In order to obtain the composites, cement masses were formulated with the addition of pure eucalyptus fibers and treated at concentrations of $2 \%, 5 \%$ and $10 \%$ by weight. The composites were characterised for water absorption, porosity, and modulus of elasticity (MOE), measuring their flexural strength at three points. A comparative analysis was done between the cement-eucayptus fiber composite and a high initial resistance Portland cement mass. The results showed that the highest valores de reforço were achieved in the cement with $2 \%$ eucalyptus fiber treated with $98 \%$ TEOS, since that cement presented the highest MOE values, presenting more

Keywords: Eucalyptus fibers. Surface treatment. Composite cement-fiber. Physical properties. Modulus of elasticity. fissure control and energy absorption after cement matrix fracture.

TESSARO, A. B.; GONÇALVES, M. R. F.; GATTO, D. A.; POLLNOW, E. N.; CARREÑO, N. L. V.; DELUCIS, R. de A. Compósitos cimentícios reforçados com fibras de eucalipto puras e tratadas com tetraetilortossilicato (TEOS $98 \%$ ). 


\section{Introdução}

A importância dos compósitos na engenharia devese ao fato de se poder alcançar um material com características superiores às convencionais, numa estratégia de produção de materiais avançados por introdução de outros materiais.

As restrições ao uso do amianto e as características frágeis e quebradiças das matrizes à base de cimento levaram ao desenvolvimento de pesquisas com materiais capazes de substituí-lo (ANJOS, 2002). Dessa maneira, surgiram estudos para a obtenção de compósitos de cimento aditivados com fibras metálicas, vegetais e sintéticas com propriedades múltiplas, tais como a capacidade de suportar cargas elevadas, controlar fissuras e absorver energia após a fratura.

No tocante aos reforços com fibras vegetais oriundas de polpa celulósica, em detrimento de sua fragilidade mecânica, quando embebidas em uma matriz de cimento, esta proporciona ao conjunto solidez e redução da propensão a fraturas (CASARIL, 2009).

Cabe salientar que o uso de fibras vegetais como reforço de matrizes tem origem na civilização antiga, com o emprego de palha ou capim na produção de tijolos de barro cru, também conhecidos como adobes. Essa prática perdeu espaço no século XX, nas décadas de 70 e 80 , com o surgimento das fibras metálicas e sintéticas (GUIMARÃES; NOVACK; BOTARO, 2010).

Os problemas causados pelo consumo de energia para a obtenção de fibras metálicas e os danos provocados ao meio ambiente pela fabricação de fibras sintéticas (por exemplo, a fibra de vidro) despertaram o interesse de pesquisadores no estudo de obtenção de reforços em matrizes cimentícias a partir da utilização das fibras vegetais, que são encontradas em abundância e obtidas por processos de baixo custo. Como exemplos citamse os trabalhos propostos por Rodrigues et al. (2013), que utilizaram fibras do bagaço da canade-açúcar e polpa de eucalipto branqueada em matriz de cimento de alta resistência inicial (CPV), para diminuir a absorção de água e a massa específica, com as características mecânicas mantidas próximas nos testes, por Correia (2011), que utilizou polpa de bambu com cimento CPV, cuja adição da fibra proporcionou maior resistência à ruptura, menos absorção de água e porosidade dos compósitos, e por Tonoli (2009), que utilizou cimento CPV e fibras de eucalipto e obteve compósitos com maior reforço na microestrutura, tendo assim ganhos nas características mecânicas.

O Brasil situa-se entre os maiores países do mundo em florestas plantadas e possui um elevado nível de extrativismo, porém ainda atua de forma desorganizada na utilização de seus recursos naturais (NASCIMENTO; PEREIRA, 2013). Enquanto em 2000 o país era responsável por 4,4\% de toda a celulose produzida no mundo, em 2009 essa razão passou para $8,7 \%$. Se considerarmos somente a celulose de mercado, ou seja, a celulose que é negociada, e não aquela que alimenta a produção de plantas de papel integradas, essa participação aumentou de $8,5 \%$ para $18,3 \%$. O Brasil saltou de uma capacidade instalada de 7,6 milhões de t/ano em 2000 para 15 milhões de t/ano em 2009, o que equivale a um crescimento médio anual de 7,7\% (ou 815 milhões de t/ano de capacidade adicional por ano). A maior parte desse crescimento veio de celulose de mercado, proveniente do eucalipto (fibra curta), destinada à exportação. Essa situação favorável da produção de celulose e o crescente consumo de materiais compostos na construção civil têm motivado o desenvolvimento de pesquisas que estudam a utilização dessas matérias-primas de origem vegetal para a adição em compósitos cimentícios (SILVA, 2013).

Para garantir a durabilidade dos compósitos reforçados com fibras vegetais, algumas técnicas de tratamento têm sido propostas, tais como impregnação com substâncias bloqueadoras e repelentes de água, selagem da matriz (diminuição da quantidade e do tamanho dos poros, bem como de sua interconectividade) e modificação da matriz por meio do uso de cimento com alto teor de alumina ou com a adição de materiais pozolânicos, do tipo sílica ativa, cinza volante ou escória (LIMA; TOLEDO FILHO, 2010). O tratamento químico das fibras através de imersão em silicato de sódio, sulfato de alumínio ou cloreto de cálcio, visando a sua impermeabilização e diminuição da absorção de água, é usado para obter ganhos de resistência mecânica dos compósitos (MAGALHÃES, 2009).

No presente trabalho apresenta-se parte de uma pesquisa envolvendo a obtenção de maior flexibilidade e elasticidade de uma matriz de cimento Portland CPV adquirida a partir da adição de fibras de eucalipto puras e tratadas com o organossilano tetraetilortossilicato TEOS $98 \%$, considerando a análise do módulo de elasticidade (MOE) obtido nos ensaios de resistência à flexão a três pontos. Não foram avaliados o módulo de ruptura e a tenacidade dos compósitos devido ao interesse da pesquisa em verificar a possibilidade do efeito reforço a partir de um maior controle de fissuras e de absorção de energia de fratura da matriz de cimento. A impregnação com TEOS

48 Tessaro, A. B.; Gonçalves, M. R. F.; Gatto, D. A.; Pollnow, E. N.; Carreño, N. L. V.; Delucis, R. de A. 
98\% resultou do interesse por sua característica de selagem (POTULSKI, 2012), que pode evitar o aumento da quantidade de água na massa e a manutenção da adequada hidratação do cimento.

\section{Materiais e métodos}

As matérias-primas empregadas no desenvolvimento deste trabalho foram cimento Portland CPV e fibras de eucalipto puras e tratadas com o organossilano tetraetilortossilicato (TEOS 98\%).

$\mathrm{O}$ cimento Portland CPV não foi caracterizado em laboratório por se tratar de um produto comercial de características normatizadas. Sua composição foi fornecida pelo fabricante (Tabela 1).

As fibras de eucalipto foram fornecidas pela empresa Celulose Riograndense, situada no município de Guaíba, RS, que utiliza o método químico de polpação Kraft para sua obtenção.

Para o desenvolvimento do trabalho as fibras de eucalipto foram tratadas de três maneiras: úmidas, a partir de dispersão em água e agitação constante, utilizando-se o agitador RoboStirr sem fio; secas em estufa com ventilação, à temperatura de $50{ }^{\circ} \mathrm{C}$ por $48 \mathrm{~h}$; e impregnadas por imersão simples em uma suspensão de TEOS $98 \%$ (especificações na Tabela 2) e álcool etílico absoluto, em agitação constante sobre um agitador magnético com aquecimento a $50{ }^{\circ} \mathrm{C}$ até a completa evaporação do álcool.

Para a obtenção dos compósitos foram formuladas massas de cimento com proporções de adição de $0 \%, 2 \%, 5 \%$ e $10 \%$ (percentual em massa) de fibras de eucalipto úmidas, secas e tratadas com TEOS $98 \%$.

Para a homogeneização das massas também se utilizou o agitador RoboStirr sem fio, e, em função do tratamento das fibras, as massas foram preparadas da seguinte forma:

(a) às fibras úmidas (em torno de $60 \%$ de umidade) inicialmente se adicionou a água e, posteriormente, o cimento, mantendo-se a agitação da mistura até a completa homogeneização;

(b) às fibras secas em estufa inicialmente se adicionou o cimento e, posteriormente, a água, mantendo-se a agitação da mistura até a completa homogeneização; e

(c) às fibras tratadas com TEOS 98\%, em constante agitação, adicionou-se o cimento e, posteriormente, a água.

Com as massas cimentícias foram conformados corpos de prova nas dimensões $127 \mathrm{~mm} \mathrm{x} \mathrm{12,7}$ $\mathrm{mm} \times$ 4,6 mm (comprimento, largura e espessura respectivamente), de acordo com a norma D 790/03 da ASTM. Esses foram caracterizados quanto à absorção de água e à porosidade aparente, de acordo com a norma ASTM C 133-97 (AMERICAN..., 2008), e quanto à resistência e flexão a três pontos, segundo a norma D 790/03 da ASTM (AMERICAN..., 2003). O ensaio de resistência à flexão foi feito em corpos de provas com idade de 28 dias, em uma máquina de ensaios universal, da marca EMIC, modelo DL 30000, com célula de carga com capacidade de $500 \mathrm{~N}$. Na massa pura de cimento foram realizados os mesmos ensaios dos compósitos cimento-fibras de eucalipto.

Tabela 1 - Composição química do cimento CPV ARI

\begin{tabular}{c|c|c|c|c|c|c|c|c|c}
\hline $\begin{array}{c}\text { Ignition } \\
\text { Ioss }\end{array}$ & $\mathrm{Al}_{2} \mathrm{O}_{3}$ & $\mathrm{SiO}_{\mathbf{2}}$ & $\mathrm{Fe}_{\mathbf{2}} \mathrm{O}_{3}$ & $\mathbf{C a O}$ & $\mathrm{MgO}$ & $\mathbf{S O}_{3}$ & $\begin{array}{c}\mathrm{CaO} \\
\text { livre }\end{array}$ & $\begin{array}{c}\text { Equiv. } \\
\text { alcal }\end{array}$ & $\begin{array}{c}\text { Resíduo } \\
\text { insolúvel }\end{array}$ \\
\hline $3,6 \%$ & $3,1 \%$ & $17,8 \%$ & $2,42 \%$ & $60,5 \%$ & $4,61 \%$ & $3,38 \%$ & $1,7 \%$ & $0,70 \%$ & $2,2 \%$ \\
\hline
\end{tabular}

Fonte: Votorantim Cimentos (2013).

Tabela 2 - Ficha Técnica do TEOS 98\%

\begin{tabular}{|c|c|c|}
\hline Sinônimos & \multicolumn{2}{|c|}{$\begin{array}{l}\text { Orthosilicic Acid Tetraethyl Ester, Silicon Tetraethoxide, Teos, } \\
\text { Tetraethoxysilane, Tetraethoxysilicon (Iv), Tetraethyl Silicate }\end{array}$} \\
\hline Fórmula linear & $\mathrm{Si}\left(\mathrm{OC}_{2} \mathrm{H}_{5}\right)_{4}$ & Fórmula estrutural \\
\hline Forma & Líquido & $\mathrm{C}_{8} \mathrm{H}_{20} \mathrm{O}_{4} \mathrm{Si}$ \\
\hline Grau de reagente & $98 \%$ & \\
\hline Peso molecular & $208,33 \mathrm{~g} / \mathrm{mol}$ & \\
\hline Ponto de ebulição & $168^{\circ} \mathrm{C}$ & $\delta_{H}$ \\
\hline
\end{tabular}

Fonte: Sigma-Aldrich Corporation (2012). 
Para avaliar o selamento da fibra ocasionado pelo tratamento com TEOS $98 \%$ foi aplicada ANOVA simples nos dados de absorção de fibra, tendo como fator o tratamento em dois níveis (fibra tratada com TEOS $98 \%$ e fibra pura). A verificação do reforço obtido pela matriz cimentícia devido à adição das fibras puras e tratadas com TEOS $98 \%$ ocorreu comparativamente à formulação de massa pura de cimento.

A fim de avaliar o efeito do teor de fibras nos compósitos obtidos foram propostos modelos matemáticos lineares e polinomiais, obtidos por meio de análises de regressão simples, utilizandose como variável independente a porcentagem de fibras, e como variáveis dependentes a absorção, a porosidade (lineares) e o módulo de elasticidade à flexão estática (polinomiais). Adicionalmente, a fim de comparar os compósitos obtidos com a massa cimentícia, bem como conferir maior exatidão à análise realizada pelos modelos matemáticos, as variáveis absorção de água, porosidade superficial e módulo de elasticidade foram analisadas em um delineamento experimental totalmente casualizado, aplicando-se para cada variável em estudo análises de variância fatorial (ANOVAs), tendo como fatores o estado da fibra em três níveis (úmida, seca e tratada com TEOS $98 \%$ ) e o teor de fibras nos três níveis $(2 \%$, $5 \%$ e $10 \%$ ). Para os casos em que a hipótese de nulidade foi rejeitada, as variáveis foram desdobradas por meio de testes de médias HSD de Tukey em 5\% de probabilidade de erro.

\section{Resultados e discussão}

\section{Caracterização das fibras de eucalipto}

A Figura 1 apresenta micrografia contendo fibras de eucalipto utilizadas na determinação das características morfológicas (comprimento e diâmetro). Para esta etapa do trabalho a amostragem foi de 36 fibras de eucalipto, e os resultados médios foram de 1.107,86 $\mu \mathrm{m}(40,56)$ para o comprimento e de $23,52 \mu \mathrm{m}(1,38)$ para o diâmetro.

Analisando a literatura, verifica-se que o comprimento médio das fibras de eucalipto $(1.107,86 \mu \mathrm{m})$ apresenta-se dentro de tamanhos mínimos, que variam entre $500 \mu \mathrm{m}$ e $3.000 \mu \mathrm{m}$ (TONOLI, 2009), e o diâmetro $(23,51 \mu \mathrm{m})$ também se apresenta nessa condição, conforme valores divulgados no estudo de Jawaid e Abdul Khail (2011), que reportam que em madeiras de espécies coníferas como o eucalipto o comprimento e o diâmetro das fibras são de cerca de $1.000 \mu \mathrm{m}$ e $20 \mu \mathrm{m}$ respectivamente. A análise morfológica indica que as fibras avaliadas no presente estudo estão dentro dos limites informados por trabalhos antecedentes.

A Tabela 3 apresenta os valores de absorção de água utilizados para a avaliação da impregnação do TEOS $98 \%$. Como se pode ver, a fibra tratada apresenta redução de $32 \%$ de absorção. Esse resultado foi muito importante para a proposta porque mostrou que ocorreu a selagem da fibra e a possibilidade de manutenção da quantidade de água necessária à hidratação do cimento.

\section{Caracterização dos compósitos cimento-fibras e da massa pura de cimento}

A Tabela 4 apresenta os modelos matemáticos propostos para predição da absorção e da porosidade dos compósitos em razão do teor de fibras, os quais foram ajustados de modo que se apresentaram estatisticamente significativos pelo teste F. Já as Figuras 2 e 3 mostram os dados brutos com suas respectivas retas de regressão.

Figura 1 - Micrografia contendo fibras de eucalipto por microscopia óptica - aumento de 10x

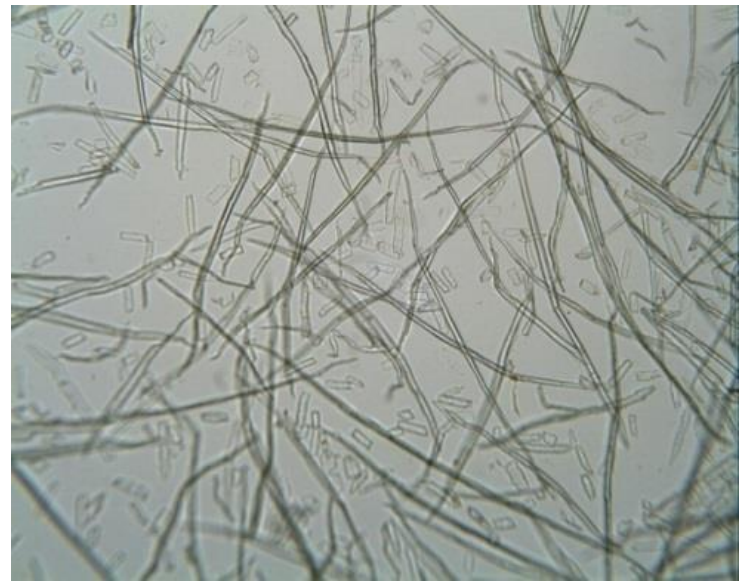

50 Tessaro, A. B.; Gonçalves, M. R. F.; Gatto, D. A.; Pollnow, E. N.; Carreño, N. L. V.; Delucis, R. de A. 
Tabela 3 - Absorção de água da fibra

\begin{tabular}{l|l|c|c}
\hline & Pura & TEOS 98\% & Teste F \\
\hline Média (\%) & 0,850 & $\begin{array}{c}0,578 \\
10,68\end{array}$ & $52,12^{*}$ \\
CV & 8,04 & 104 & \\
\hline
\end{tabular}

Nota: Legenda:

CV - coeficiente de variação; e

${ }^{*}=$ significativo em $5 \%$ de probabilidade de erro, de acordo com o teste HSD de Tukey.

Tabela 4 - Modelos matemáticos propostos para a avaliação do efeito do teor de fibras na absorção e porosidade dos compósitos cimento-fibras

\begin{tabular}{l|l|c|c|c}
\hline & Equação & $\mathbf{R}^{2}$ & Syx & Valor F \\
\hline \multicolumn{5}{c}{ Absorção } \\
\hline Seca & Abs $=0,108458+0,0166373 \cdot \%$ fib & 0,90 & 0,02273 & $91,21^{* *}$ \\
Úmida & Abs $=0,131938+0,0201322 \cdot \%$ fib & 0,81 & 0,03977 & $43,61 * *$ \\
TEOS 98\% & Abs $=0,0949046+0,011395 \cdot \%$ fib & 0,86 & 0,01833 & $65,79 * *$ \\
\hline \multicolumn{5}{c}{ Porosidade } \\
\hline Seca & Po $=0,139148+0,0103965 \cdot \%$ fib & 0,81 & 0,02065 & $43,15^{* *}$ \\
Úmida & Po $=0,133656+0,00835536 \cdot \%$ fib & 0,89 & 0,01185 & $84,52 * *$ \\
TEOS 98\% & Po $=0,121483+0,00396476 \cdot \%$ fib & 0,46 & 0,01757 & $8,66 *$ \\
\hline
\end{tabular}

Nota: Legenda:

$$
\begin{aligned}
& \text { Abs = absorção do compósito (\%); } \\
& \text { Po = porosidade (\%); } \\
& \% \text { fib = porcentagem de fibra (\%); } \\
& \mathrm{R}^{2}=\text { coeficiente de determinação; } \\
& \text { Syx = erro médio padrão; } \\
& \mathrm{F}=\text { valor de } \mathrm{F} \text { calculado; } \\
& { }^{*}=\text { significativo a } 5 \% \text { de probabilidade de erro; e } \\
& { }^{* *}=\text { significativo a } 1 \% \text { de probabilidade de erro. }
\end{aligned}
$$

Figura 2 - Valores de absorção de água dos compósitos obtidos em função do teor de fibras de eucalipto em corpos de prova com 28 dias de idade

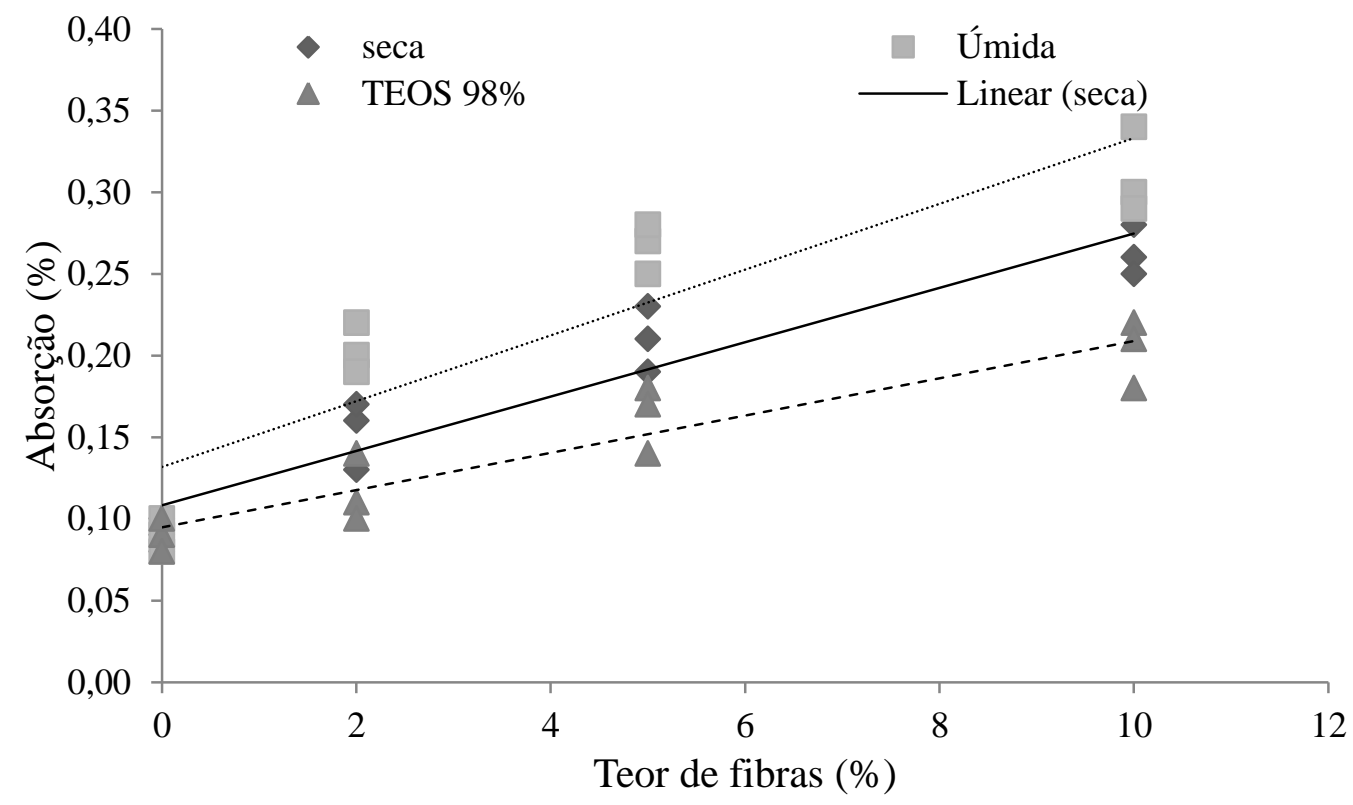


Figura 3 - Valores de porosidade aparente dos compósitos obtidos em função do teor de fibras de eucalipto em corpos de prova com 28 dias de idade

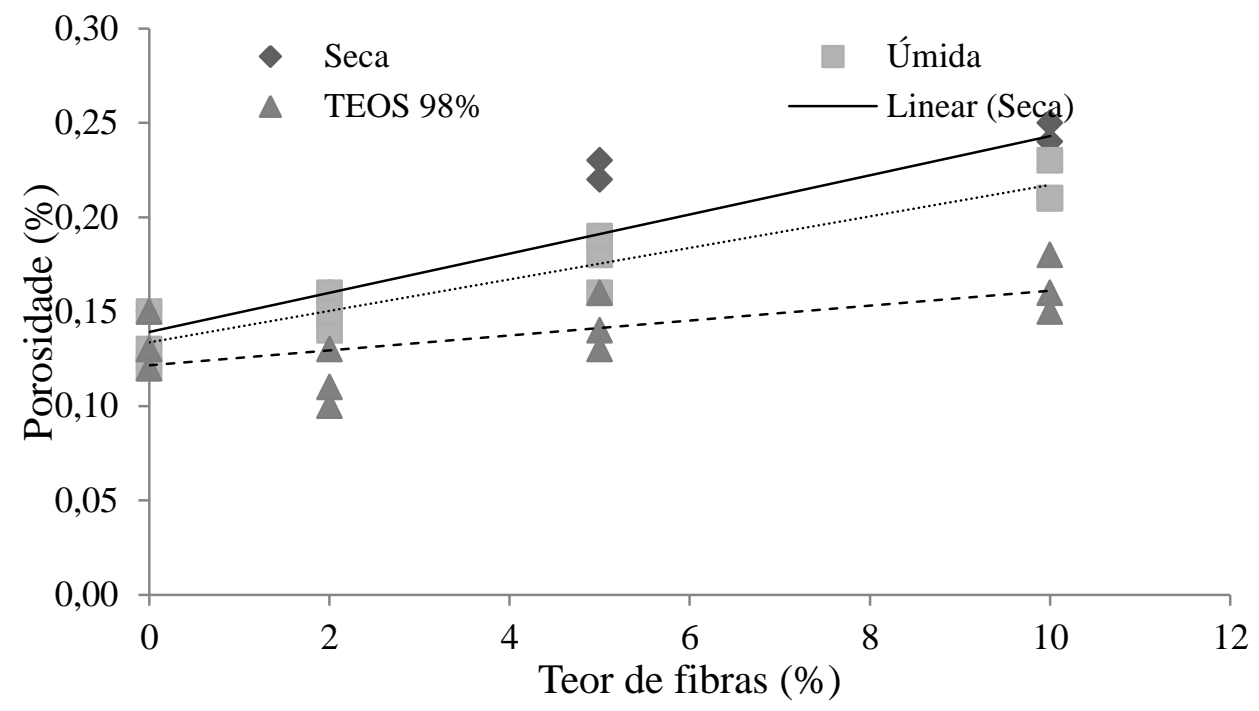

Verifica-se pelos modelos ajustados na Tabela 4 que o tratamento estatístico empregado mostrou um bom nível de exatidão na avaliação do efeito do teor de fibras nas propriedades físicas de absorção e porosidade dos compósitos obtidos, na medida em que até $90 \%$ da variabilidade dos dados foi explicada em razão das equações de regressão.

Como se pode ver nas Figuras 2 e 3, verifica-se que o compósito com fibras de eucalipto tratadas com TEOS $98 \%$ é o que apresenta os menores valores de absorção de água e porosidade aparente. Esse resultado comprova que a impregnação com TEOS $98 \%$ interfere positivamente no desempenho do compósito. Além disso, também se observa que o aumento da quantidade de fibras, independentemente do tratamento, resulta em maiores valores de absorção de água e porosidade aparente.

Na Tabela 5 estão dispostos os modelos matemáticos propostos para a predição do módulo de elasticidade dos compósitos a partir de seu teor de fibras. Adicionalmente, a Figura 4 apresenta os dados brutos de MOE obtidos, dotados de suas respectivas curvas de descrição de comportamento.

Como se pode ver na Figura 4, independentemente do tratamento e da quantidade de fibras, comparativamente os maiores valores de $\mathrm{MOE}$ ocorreram para os compósitos com $2 \%$ de fibra. Valores de teor de fibras superiores a $2 \%$ resultaram em perda de resistência mecânica. Para essas situações o MOE perdeu significância em função do aumento observado na porosidade do compósito (aspecto comprovado na Figura 3), possivelmente causado pela maior concentração de fibras e pelo aumento de vazios sem preenchimento.

$\mathrm{Na}$ análise multifatorial o fator tratamento foi o único que apresentou efeito significativo nos compósitos.

As propriedades físicas e mecânicas da massa pura de cimento são apresentadas na Tabela 6. Os valores representam a média de 10 corpos de prova. Para a massa pura não foi aplicado tratamento estatístico.

Verificou-se para modelos ajustados para a predição do MOE que, embora sua precisão de ajuste tenha sido menor do que a dos modelos lineares que avaliaram a absorção e a porosidade, eles são considerados válidos na medida em que lograram significância estatística.

Para fins de comparação com a massa pura de cimento, elaborou-se a Tabela 7, que apresenta os valores médios de absorção de água, porosidade aparente e MOE. Para tal, as ANOVAs fatoriais empregadas definiram que, para a absorção de água, foram significativos os efeitos dos fatores tratamento $(\mathrm{F}=80,04 ; \mathrm{p}<0,01)$ e teor $(\mathrm{F}=92,37$; $\mathrm{p}<0,01)$ de fibras; já a interação entre os fatores não foi significativa $(\mathrm{F}=0,81 ; \mathrm{p}>0,05)$. Analogamente, para a porosidade superficial, o tratamento $(\mathrm{F}=32,60 ; \mathrm{p}<0,01)$ e o teor $(\mathrm{F}=41,25$; $\mathrm{p}<0,01)$ de fibras foram fatores com efeito significativo, já a interação entre os mesmos não logrou ser significava $(F=1,00 ; p>0,05)$. Por outro lado, o módulo de elasticidade foi afetado significativamente pelos fatores tratamento $(\mathrm{F}=13,86 ; \mathrm{p}<0,01)$ e teor $(\mathrm{F}=57,04 ; \mathrm{p}<0,01)$ de fibras, bem como pela interação entre eles, a qual também foi significativa $(\mathrm{F}=6,84 ; \mathrm{p}<0,01)$. 
Tabela 5 - Modelos matemáticos propostos para a avaliação do efeito do teor de fibras no módulo de elasticidade ao ensaio de flexão estática dos compósitos cimento-fibras

\begin{tabular}{|c|c|c|c|c|}
\hline & Equação & $\mathbf{R}^{2}$ & Syx & Valor F \\
\hline Seca & $\mathrm{MOE}=11.556,9+497,499 . \%$ fib $-63,0383 . \% \mathrm{fib}^{\wedge} 2$ & 0,19 & $1.904,05$ & $3,88^{*}$ \\
\hline Úmida & $\mathrm{MOE}=11.076,8+1.212,15 . \%$ fib $-167,418 . \% \mathrm{fib}^{\wedge} 2$ & 0,71 & $1.750,4$ & $40,68 * *$ \\
\hline TEOS & $\mathrm{MOE}=11.364,0+1.187,87 . \%$ fib $-117,549 . \% \mathrm{fib}^{\wedge} 2$ & 0,30 & $2.002,97$ & $7,09 * *$ \\
\hline
\end{tabular}

Figura 4 - Valores do módulo de elasticidade (MOE) dos compósitos obtidos em função do teor de fibras de eucalipto em corpos de prova com 28 dias de idade

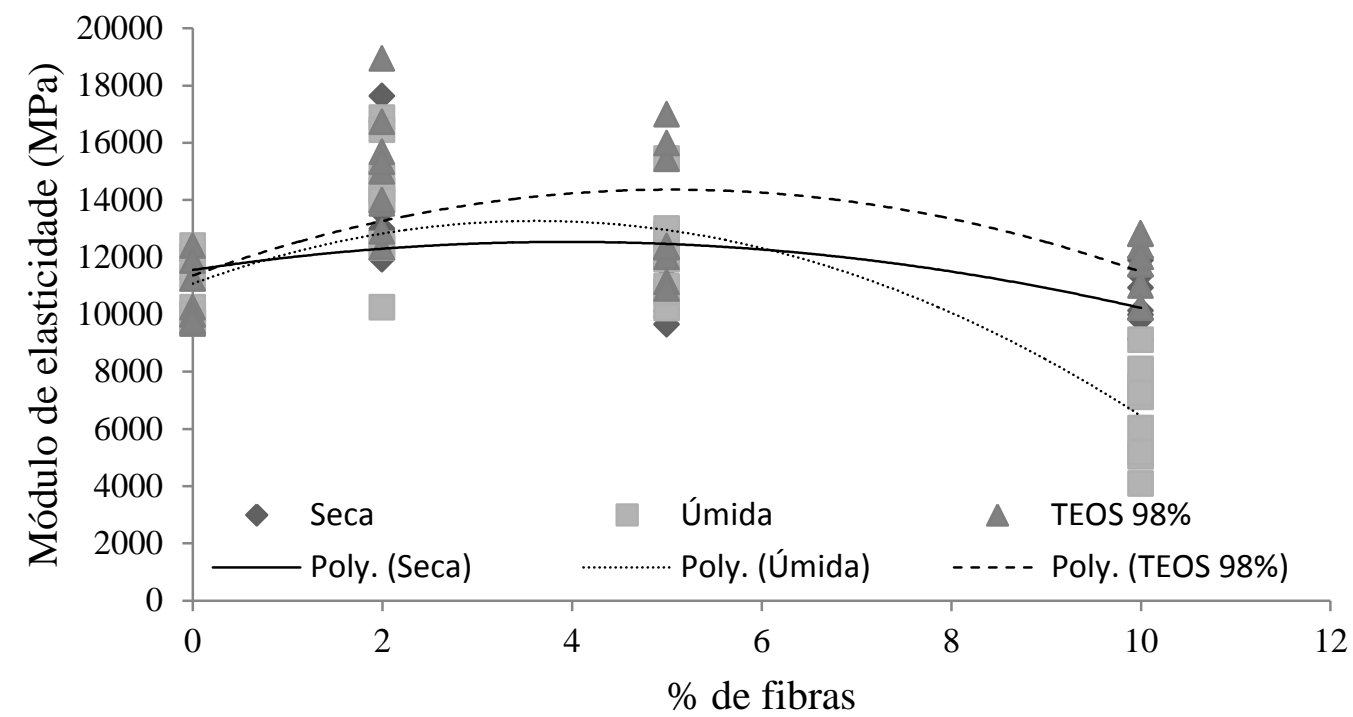

Tabela 6 - Características da massa pura de cimento CPV ARI

\begin{tabular}{l|l|r}
\hline \multicolumn{1}{c|}{ Propriedades } & \multicolumn{1}{c|}{ Média } & \multicolumn{1}{c}{ CV } \\
\hline Absorção de água (\%) & 0,176 & 11,11 \\
Porosidade aparente (\%) & 0,130 & 11,54 \\
Módulo de elasticidade - MOE (MPa) & $10.546,85$ & 9,69 \\
\hline
\end{tabular}

Tabela 7 - Características físicas e mecânicas dos compósitos de cimento-fibras de eucalipto

\begin{tabular}{|c|c|c|c|c|}
\hline & 2 & 5 & 10 & Média \\
\hline \multicolumn{5}{|c|}{ Absorção de água (\%) } \\
\hline Úmida & $0,193_{(7,51)} \mathrm{A} \mathrm{b}$ & $0,248_{(5,72)} \mathrm{B} \mathrm{b}$ & $0,310_{(8,53)} \mathrm{B} \mathrm{b}$ & $0,250_{(19,03)} \mathrm{c}$ \\
\hline Seca & $0,135_{(13,57)} \mathrm{A} \mathrm{a}$ & $0,195_{(9,52)} \mathrm{B}$ a & $0,250_{(5,80)} \mathrm{Cb}$ & $0,1933_{(24,10)} b$ \\
\hline TEOS $98 \%$ & $0,108_{(17,84)} \mathrm{A} \mathrm{a}$ & $0,153_{(12,74)} \mathrm{AB}$ a & $0,195_{(10,23)} \mathrm{B}$ a & $0,152_{(25,86)} \mathrm{a}$ \\
\hline Média & $0,145_{(26,08)} \mathrm{A}$ & $0,198_{(22,35)} \mathrm{B}$ & $0,251_{(19,25)} \mathrm{C}$ & $0,210_{(29,20)}$ \\
\hline \multicolumn{5}{|c|}{ Porosidade aparente (\%) } \\
\hline Úmida & $0,156_{(6,66)} \mathrm{A} \mathrm{b}$ & $0,195_{(8,64)} \mathrm{A} a b$ & $0,233_{(5,32)} \mathrm{B} \mathrm{b}$ & $0,195_{(18,11)} b$ \\
\hline Seca & $0,173_{(3,76)} \mathrm{A} \mathrm{b}$ & $0,228_{(9,75)} \mathrm{B} \mathrm{b}$ & $0,265_{(8,92)} \mathrm{B} \mathrm{b}$ & $0,222_{(19,52)} \mathrm{c}$ \\
\hline TEOS $98 \%$ & $0,128_{(13,47)} \mathrm{A} \mathrm{a}$ & $0,160_{(10,65)} \mathrm{AB}$ a & $0,181_{(9,35)} \mathrm{B} \mathrm{a}$ & $0,156_{(18,21)} \mathrm{a}$ \\
\hline Média & $0,153_{(15,46)} \mathrm{A}$ & $0,194_{(19,02)} \mathrm{B}$ & $0,226_{(16,96)} \mathrm{C}$ & $0,173(23,14)$ \\
\hline \multicolumn{5}{|c|}{ Módulo de Elasticidade - MOE (MPa) } \\
\hline Úmida & $13935,5_{(14,84)} \mathrm{B}$ a & $12245,5_{(12,69)} \mathrm{B}$ ab & $6588,91_{(24,6)} \mathrm{A} \mathrm{a}$ & $10923,3_{(33,15)} \mathrm{a}$ \\
\hline Seca & $14403,9{ }_{(12,91)} \mathrm{A} a$ & $11121,7{ }_{(8,99)} \mathrm{A} \mathrm{a}$ & $10480,5{ }_{(10,49)} \mathrm{A} \mathrm{b}$ & $12002,0_{(18,27)} \mathrm{a}$ \\
\hline TEOS 98\% & $14971,8_{(13,52)} \mathrm{B} \mathrm{a}$ & $13275,1_{(16,89)} \mathrm{AB} b$ & $11692,0_{(8,48)} \mathrm{A} \mathrm{b}$ & $13313,0_{(16,75)} b$ \\
\hline Média & $14437,0_{(13,55)} \mathrm{C}$ & $12214,1_{(15,10)} \mathrm{B}$ & $9587,15_{(26,39)} \mathrm{A}$ & $12079,4_{(24,00)}$ \\
\hline
\end{tabular}

Nota: médias seguidas por letras iguais maiúsculas nas linhas e minúsculas nas colunas não são diferentes estatisticamente de acordo com o teste HSD de Tukey em $5 \%$ de probabilidade de erro. 
Figura 5 - MEV de uma fibra de eucalipto. Poros presentes na fibra. Aumento de $2.000 x$

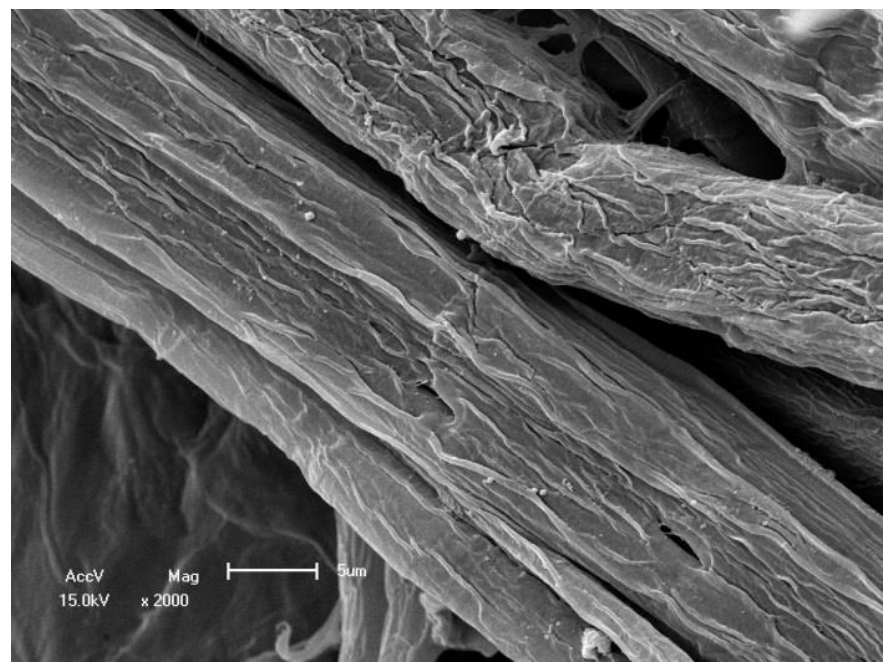

Os resultados apresentados na Tabela 7 ratificam os comportamentos evidenciados e descritos nos gráficos ajustados nas Figuras 2, 3 e 4. Contudo, essas comparações se mostram importantes à medida que os testes de médias realizados na Tabela 7 evidentemente conferem maior confiabilidade para comparações múltiplas como as realizadas no presente estudo.

Quando comparados à massa pura de cimento, todos os compósitos obtidos apresentaram-se com valores maiores para a absorção de água e porosidade aparente (Tabela 7). Esse resultado se justifica, provavelmente, pela presença da fibra, que possui porosidade intrínseca como demonstrado na Figura 5.

\section{Conclusões}

A partir dos resultados obtidos pode-se inferir as seguintes conclusões:

(a) é possível obter compósitos de cimento aditivados com fibras de eucalipto puras e tratadas com TEOS $98 \%$;

(b) o emprego do TEOS $98 \%$ alterou as propriedades de absorção de água, porosidade aparente e MOE dos compósitos obtidos;

(c) o compósito com $2 \%$ de fibras de eucalipto tratadas com TEOS $98 \%$ foi o que apresentou o maior reforço no tocante à flexibilidade e à elasticidade da matriz de cimento; e

(d) o aumento da percentagem de fibras, independentemente do preparo (úmida, seca ou tratada com TEOS 98\%) resultou em menor flexibilidade e elasticidade da matriz de cimento.

\section{Referências}

ANJOS, M. A. S. Compósito à Base de Cimento Reforçado Com Polpa de Bambu: caracterização física, mecânica e microestrutural. Rio de Janeiro, 2002. 96 f. Dissertação (Mestrado em Engenharia Civil) - Pontifícia Universidade Católica, Rio de Janeiro, 2002.

\section{AMERICAN SOCIETY FOR TESTING AND}

MATERIALS. ASTM C - 133/97: standard test methods for cold crushing strength and modulus of rupture of refractories. Philadelphia, 2008.

\section{AMERICAN SOCIETY FOR TESTING AND} MATERIALS. ASTM D 790-03: standard test methods for flexural properties of unreinforced and reinforced plastics and electrical insulating materials. Philadelphia, 2003.

\section{CASARIL, A. Modelagem Matemática e} Avaliação Experimental do Módulo de Elasticidade de Materiais Compósitos Particulados de Matriz Fenólica Para Uso Como Material de Fricção. Porto Alegre, 2009. 67 f. Dissertação (Mestrado em Engenharia de Minas, Metalúrgica e de Materiais) - Universidade Federal do Rio Grande do Sul, Porto Alegre, 2009.

CORREIA, V. C. Produção e Caracterização de Polpa Organossolve de Bambu Para Reforço de Matrizes Cimentícias. Pirassununga, 2011. $121 \mathrm{f}$. Dissertação (Mestrado em Zootecnia) Universidade de São Paulo, Pirassununga, 2011.

GUIMARÃES, M.; NOVACK, K. M.; BOTARO, V. R. Caracterização Anatômica da Fibra de Bambu (Bambusavulgaris) Visando Sua Utilização em Compósitos Poliméricos. Revista

Iberoamericana de Polímeros, v. 11, n. 7, p. 442 456, 2010. 
JAWAID, M.; ABDUL KHAIL, H. P. S. Cellulosic/Synthetic Fibre Reinforced Polymer Hybrid Composites: a review. Carbohydrate Polymers, v. 86, n. 1, p. 1-18, 2011.

LIMA, P. R. L.; TOLEDO FILHO, R. D. Uso de Metacaulinita Para Incremento da Durabilidade de Compósitos à Base de Cimento Reforçados Com Fibras de Sisal. Ambiente Construído, Porto Alegre, v. 8, n. 4, p. 7-19, out./dez. 2010.

MAGAlHÃES, A. C. T. V. Estudos de Fibras

Vegetais, Mucilagem de Cacto e Gesso em Componentes Construtivos. Brasília, 2009. 123 f. Dissertação (Mestrado em Arquitetura e Urbanismo) - Escola de Arquitetura, Universidade de Brasília, Brasília, 2009.

NASCIMENTO, R. M.; PEREIRA, F. S. C. Florestal Brasileiro. Brasília, SFB, 2013.

POTULSKI, D. C. Efeito da Incorporação de Microfibrilas de Celulose Sobre as Propriedades do Papel. Curitiba, 2012. 74 f. Dissertação (Mestrado em Engenharia Florestal) Escola de Engenharia, Universidade Federal do Paraná, Curitiba, 2012.

RODRIGUES, M. S. et al. Cinza de Palha de Cana-de-Açúcar Como Adição Mineral em Fibrocimentos. Revista Brasileira de Engenharia Agrícola e Ambiental, v. 17, n. 12, p. 1347-1354, 2013.
SIGMA-ALDRICH CORPORATION. [Catálogo]. Disponível em:

<http://www.sigmaaldrich.com/catalog/product/ald rich/131903?lang=en\&region=US $>$. Acesso em: 21 set. 2012.

SILVA, M. F. Engenharia de Superfície de Nanopartículas Magnéticas Para Biomedicina: recobrimento com macromoléculas visando estabilização e compatibilidade em meio fisiológico. São Paulo, 2013. 90 f. Dissertação (Mestrado em Química) - Escola de Química, Universidade de São Paulo, São Paulo, 2013.

TONOLI, G. H. D. Fibras Curtas de Eucalipto Para Novas Tecnologias em Fibrocimento. São Paulo, 2009. 150 f. Tese (Doutorado em Ciência e Engenharia de Materiais) - Universidade de São Paulo, São Paulo, 2009.

\section{VOTORANTIM CIMENTOS. Cimento: CP V} ARI. Disponível em:

<http://www.mapadaobra.com.br/produtos/ciment o/linha/cimento-cp-v-ari>. Acesso em: 24 out. 2013.

\section{Agradecimentos}

À CAPES e à FAPERGS.

\footnotetext{
Alessandra Buss Tessaro

Programa de Pós-Graduação em Ciência e Engenharia de Materiais | Universidade Federal de Pelotas | Rua Felix da Cunha, 809, Campus Universitário | Caixa Postal 354 | Pelotas - RS - Brasil | CEP 96010-000 | Tel.: (53) 3228-3705 | E-mail: alessandrabuss@gmail.com

Margarete Regina Freitas Gonçalves

Programa de Pós-Graduação em Ciência e Engenharia de Materiais | Universidade Federal de Pelotas | E-mail: margareterfg@gmail.com

Darci Alberto Gatto

Programa de Pós-Graduação em Ciência e Engenharia de Materiais | Universidade Federal de Pelotas | E-mail: darcigatto@yahoo.com.br

Edilson Nunes Pollnow

Escola de Engenharia, Conselho de Ensino, Pesquisa e Extensão | Universidade Federal do Rio Grande | Av. Itália km 8 Bairro Carreiros, Campus Carreiros | Rio Grande - RS - Brasil | CEP 96203-900 | Tel.: (53) 3293-5209 | E-mail: edilson.pollnow@hotmail.com

Neftali Lenin Villarreal Carreño

Programa de Pós-Graduação em Ciência e Engenharia de Materiais | Universidade Federal de Pelotas | E-mail: nlv.carreno@gmail.com

Rafael de Ávila Delucis

Engenharia Industrial Madeireira | Universidade Federal de Pelotas | Rua Conde de Porto Alegre, 793, Centro | Pelotas - RS - Brasil | CEP 96010-290 | Tel.: (53) 3921-1265 | E-mail: r.delucis@hotmail.com
}

Revista Ambiente Construído

Associação Nacional de Tecnologia do Ambiente Construído

Av. Osvaldo Aranha, $99-3^{\circ}$ andar, Centro

Porto Alegre - RS - Brasil CEP $90035-190$

Telefone: +55 (51) 3308-4084

Fax: +55 (51) 3308-4054

www.seer.ufrgs.br/ambienteconstruido

E-mail: ambienteconstruido@ufrgs.br 